\title{
Reflexion einer Bildung für nachhaltige Entwicklung aus bildungstheoretischer Perspektive
}

\author{
Marco Rieckmann \\ Universität Vechta
}

Kontakt: marco.rieckmann@uni-vechta.de

eingereicht: 04.06.2021; überarbeitet: 07.09.2021; angenommen: 21.09.2021

\begin{abstract}
Zusammenfassung: Bildung für nachhaltige Entwicklung (BNE) ist ein relativ junges Bildungskonzept, das sich in der Erziehungswissenschaft wie auch in der pädagogischen Praxis in den letzten 20 Jahren etabliert hat. Dieser Artikel reflektiert das Konzept einer BNE aus bildungstheoretischer Perspektive und beleuchtet Anschlussperspektiven zwischen BNE und ausgewählten bildungstheoretischen Positionen und pädagogischen Strömungen. Mit den Ausführungen in diesem Artikel wird deutlich, dass BNE sich erstens besser verstehen und verorten lässt, wenn sie auf Theorien der Erziehung und Bildung Bezug nimmt. Zweitens lässt sich BNE als eine aktualisierte Konzeption von Allgemeinbildung im Sinne Klafkis betrachten. Drittens sind Theorien zur transformativen Bildung sehr nützlich, um die im Rahmen einer BNE angestrebte Werte-Bildung zu beschreiben. Viertens ergeben sich aus Krügers Reflexiver Erziehungswissenschaft Anregungen für eine Auseinandersetzung mit der Notwendigkeit struktureller und institutioneller Transformation und für eine inklusivere Gestaltung von BNE. Fünftens weist BNE Parallelen zu Freires Befreiungspädagogik auf, und sie könnte von einer Auseinandersetzung mit den didaktischen Zugängen der Befreiungspädagogik profitieren. Mit dem Artikel wird ein Beitrag dazu geleistet, zum einen BNE stärker in der Erziehungswissenschaft zu verorten und zum anderen die Anschlussfähigkeit an allgemeine erziehungswissenschaftliche Diskurse herzustellen.

Schlagwörter: Bildung für nachhaltige Entwicklung, Bildungstheorie, Klafki

Abstract: Education for Sustainable Development (ESD) is a relatively young educational concept that has established itself in educational research as well as in educational practice in the last 20 years. This paper reflects on the concept of ESD from an educational theory perspective and sheds light on connecting perspectives between ESD and selected educational theory positions and pedagogical currents. With the discussion in this paper, it becomes clear that, firstly, ESD can be better understood and located if it refers to theories of education. Secondly, ESD can be seen as an updated conception of general education in Klafki's sense. Thirdly, theories of transformative education are very useful in describing the value education sought in ESD. Fourthly, Krüger's reflexive educational science gives rise to suggestions for addressing the need for structural and institutional transformation and for an inclusive design of ESD. Fifthly, ESD has parallels to Freire's liberation pedagogy, and it could benefit from an engagement with the didactic approaches of liberation pedagogy. The paper contributes to locating ESD more strongly in educational research and to establishing a connection to general educational discourses.
\end{abstract}

Keywords: Education for sustainable development, education theory, Klafki

\section{Einführung}

Vor dem Hintergrund der globalen Umweltkrise, Armut und Ungerechtigkeit (World Bank, 2020; UN Environment, 2019; Steffen et al., 2015) und im Hinblick auf das Erreichen der Nachhaltigkeitsziele der Vereinten Nationen (Sustainable Development Goals - SDGs) (United Nations, 2015) kommt Bildung die Aufgabe zu, die Lernenden zu befähigen, globale Zusammenhänge zu verstehen und aktiv an der nachhaltigen „Großen Transformation“ (WBGU, 2011) der Gesellschaft mitzuwirken, was auch die Befähigung der Lernenden zur Veränderung gesellschaftlicher Strukturen einschließt (Rieckmann, 2020; UNESCO, 
2017). Das Bildungskonzept der Bildung für nachhaltige Entwicklung (BNE) zielt darauf ab, diesem Anspruch gerecht zu werden. Seit Ende der 1990er-Jahre wird in der bildungswissenschaftlichen Diskussion sowie der Bildungspraxis verstärkt Bezug auf eine nachhaltige Entwicklung genommen. In diesem Kontext ist das Konzept einer BNE entwickelt worden (Rieckmann, 2016; Michelsen \& Fischer, 2015).

BNE hat sich in den letzten 20 Jahren als ein eigenständiges (interdisziplinäres) Forschungsfeld etabliert (Barth, 2016; Gräsel et al., 2012) und die Implementierung einer BNE ist in der Bildungspraxis durch verschiedene Modellprojekte und vor allem aber auch durch internationale Programme (UN-Dekade BNE, Weltaktionsprogramm BNE, Programm ,BNE für 2030') gefördert worden (Rieckmann, 2016; Michelsen \& Fischer, 2015). Bisher sind die Parallelen und Bezüge von BNE zu erziehungswissenschaftlichen Theorien zu Bildung und Erziehung jedoch relativ wenig betrachtet worden (zum Bildungsbegriff der BNE: Müller, 2000; zur Funktion von Lernen, Erziehung und Bildung in der Weltgesellschaft: Seitz, 2002; zu Perspektiven religiöser Bildung in der Weltgesellschaft: Simojoki, 2012).

Vor diesem Hintergrund reflektiert dieser Artikel das Konzept einer BNE aus bildungstheoretischer Perspektive und beleuchtet Anschlussperspektiven zwischen BNE und ausgewählten bildungstheoretischen Positionen und pädagogischen Strömungen. Dazu wird BNE im Folgenden zu Klafkis Bildungstheorie, zur transformativen Bildung nach Koller, zu Krügers Reflexiver Erziehungswissenschaft und Freires Befreiungspädagogik ins Verhältnis gesetzt. Damit sind für diese Reflexion bildungstheoretische Positionen und pädagogische Strömungen ausgewählt worden, die - wie sich zeigen wird - große Parallelen mit BNE bzw. Potential für die theoretische Fundierung und Weiterentwicklung einer BNE aufweisen. Es soll ein Beitrag dazu geleistet werden, zum einen BNE stärker in der Erziehungswissenschaft zu verorten und zum anderen die Anschlussfähigkeit an allgemeine erziehungswissenschaftliche Diskurse herzustellen. ${ }^{1}$

\section{Bildung für nachhaltige Entwicklung im Spannungsfeld zwischen Erziehung und Bildung}

Im internationalen erziehungswissenschaftlichen Diskurs lassen sich zwei Strömungen einer BNE (Education for Sustainable Development - ESD) voneinander abgrenzen: ESD 1 und ESD 2 (Vare \& Scott, 2007). Diese Unterscheidung findet sich auch bei Wals (2011), der von einem „instrumental approach“ und einem „emancipatory approach“ spricht.

ESD 1 geht von der Überzeugung aus, dass es bestimmte Werte und Verhaltensweisen gibt, die eindeutig mit einer nachhaltigen Entwicklung verbunden sind. Expert*innen können diese identifizieren. Ziel von BNE ist es dementsprechend, Bewusstsein für eine nachhaltige Entwicklung zu schaffen, mit Nachhaltigkeit verbundene Werte zu vermitteln und als nachhaltig erkannte Verhaltensweisen zu fördern. Es sollen durch die Bildungsprozesse bestimmte Verhaltensweisen wie die Trennung von Müll oder das Sparen von Energie befördert werden (Vare \& Scott, 2007). Unter Bezug auf Ansätze der Umweltpsychologie (Wals, 2011) werden z. B. Anreize gegeben, um ein bestimmtes, vermeintlich nachhaltiges Verhalten attraktiv zu machen.

Dieser Ansatz einer BNE im Sinne von ESD 1 wird z. B. in der kürzlich bei der UNESCO-Weltkonferenz verabschiedeten ,Berliner Erklärung zur Bildung für nachhaltige Entwicklung' deutlich: „[...] dabei beim Einzelnen eine Verhaltensänderung für nachhaltige Entwicklung, Chancengleichheit und Achtung der Menschenrechte sowie einen grundlegenden strukturellen und kulturellen Wandel auf Systemebene in Wirtschaft und Gesellschaft zu fördern und auch die notwendigen politischen Maßnahmen für diesen Wandel voranzutreiben“ (UNESCO, 2021, Absatz 6c).

\footnotetext{
${ }^{1}$ Ich danke Vertr. Prof.in Dr. Sarah Gaubitz (Universität Siegen) sowie zwei anonymen Gutachter*innen für wertvolle Hinweise zu diesem Beitrag.
} 
Aber auch in Stellungnahmen von Regierungen und Nicht-Regierungsorganisationen wird häufig Bezug darauf genommen, dass von einer BNE direkte Beiträge zu einer nachhaltigeren Lebensweise erwartet werden.

ESD 2 hingegen geht von einem reflexiveren Ansatz aus. Hier geht es weniger darum, Denk- oder Verhaltensweisen vorzugeben, sondern Individuen sollen in die Lage versetzt werden, selbst über Fragen einer nachhaltigen Entwicklung nachzudenken und ihre eigenen Antworten zu finden (Vare \& Scott, 2007). In diesem Zusammenhang wird nachhaltige Entwicklung nicht als ein geschlossener ExpertenDiskurs, sondern als ein offener gesellschaftlicher (Lern-)Prozess gesehen. Leitend ist dabei die Erkenntnis, dass oft gar nicht sicher ist, welche Verhaltensweisen effektiv die nachhaltigeren sind (Wals, 2011). „In ESD 2, we can't measure success in terms of environmental impacts because this is an open-ended process; outcomes will depend on people's unforeseen decisions in future, unforeseeable circumstances. But we can research the extent to which people have been informed and motivated, and been enabled to think critically and feel empowered to take responsibility" (Vare \& Scott, 2007, S. 194).

BNE im Sinne von ESD 2 befähigt zu einer kritischen Auseinandersetzung mit einer nachhaltigen Entwicklung und mit der Komplexität, der Unsicherheit sowie den Widersprüchen, die mit ihr verbunden sind. Hier wird eine nachhaltige Entwicklung selbst als ein Lernprozess verstanden (Vare \& Scott, 2007). Im Fokus stehen Kompetenzentwicklung und kritisches Denken als Voraussetzungen für das Treffen von eigenen Entscheidungen im Sinne einer nachhaltigen Entwicklung (Mulder, 2014; Wals, 2011). Tabelle 1 gibt einen Überblick über die beiden Strömungen.

\begin{tabular}{|c|c|}
\hline \multirow[t]{3}{*}{ ESD 1} & - Promoting/facilitating changes in what we do \\
\hline & $\begin{array}{l}\text { - Promoting (informed, skilled) behaviours and ways of thinking, where the need } \\
\text { for this is clearly identified and agreed }\end{array}$ \\
\hline & - Learning for sustainable development \\
\hline \multirow[t]{3}{*}{ ESD 2} & $\begin{array}{l}\text { - Building capacity to think critically about (and beyond) what experts say and to } \\
\text { test sustainable development ideas }\end{array}$ \\
\hline & - Exploring the contradictions inherent in sustainable living \\
\hline & - Learning as sustainable development \\
\hline
\end{tabular}

Tab. 1: ESD 1 und ESD 2 (Vare \& Scott, 2007, S. 193f.)

Setzt man diese Überlegungen zu den zwei Strömungen einer BNE in Beziehung zum deutschsprachigen Diskurs über die verschiedenen Säulen der Pädagogik (Sozialisation, Erziehung, Bildung, Lernen; Stein, 2017), lässt sich ESD 1 als Erziehung und ESD 2 als Bildung verstehen.

Denn mit Erziehung werden Handlungen beschrieben, „durch die Menschen versuchen, das Gefüge der psychischen Disposition anderer Menschen in irgendeiner Hinsicht dauerhaft zu verbessern und seine als wertvoll beurteilten Bestandteile zu erhalten oder die Entstehung von Dispositionen, die als schlecht bewertet werden, zu verhüten“ (Brezinka, 1990, S. 95). So stellt also die direkte, intentionale Förderung einer - vermeintlich - nachhaltigen Verhaltensweise eine Erziehungsmaßname dar, die durch ein klares Hierarchiegefälle zwischen den Erziehenden und der*dem zu Erziehenden charakterisiert ist (Stein, 2017). Hingegen „,sich kompetent und adäquat mit Schlüsselproblemen der Menschheit auseinanderzusetzen“ (Stein, 2017, S. 66) zu können, ist ein Merkmal von Bildung.

Erziehungs- und Bildungsprozesse sind beide Teile der Sozialisation des Individuums und hängen eng zusammen (Stein, 2017). Im Einklang damit betrachten auch Vare \& Scott (2007) ESD 1 und ESD 2 als komplementär. Sie favorisieren allerdings doch ESD 2 - so wie auch Wals (2011) sich für den von ihm als „emancipatory approach“ bezeichneten Ansatz ausspricht. Diese Argumentation findet sich auch 
bei de Haan: BNE beziehe sich nicht auf die Ziele, Zwecke und Absichten des Handelns von Individuen, sondern auf die Wirkungen. Sie solle Individuen in die Lage versetzen, „wenn sie entsprechende Ziele, Zwecke oder Absichten haben“" im Sinne einer nachhaltigen Entwicklung handeln zu können (de Haan et al., 2008, S. 117). Es gehe mithin um die „Eröffnung von Möglichkeiten“ (de Haan et al., 2008, S. 123) und nicht darum, zu einem bestimmten vermeintlich nachhaltigkeitskonformen Verhalten zu erziehen.

Während dieser Aussage vor allem in Bezug auf Hochschulbildung und Erwachsenenbildung sowie zu hohem Maße auch für die schulische Bildung zuzustimmen ist, sollte nicht außer Acht gelassen werden, dass insbesondere der frühkindlichen Bildung, aber auch durchaus der Schule auch ein Erziehungsauftrag zukommt. Oder um es mit Blick auf individuelle Lern- und darauf aufbauende Sozialisationsprozesse zu sagen: Im Kindesalter sollte ESD 1, also BNE im Sinne von Erziehung, durchaus eine Rolle spielen, wenn es z. B. um die Förderung von sachgemäßer Mülltrennung und -entsorgung oder dem Entgegenwirken diskriminierenden Verhaltens - im Sinne des Anti-Bias-Ansatzes - (Gramelt, 2010) geht. Gleichzeitig ist aber auch ESD 2, also BNE im Sinne von Bildung, in Kindergärten und Grundschulen zu realisieren.

Bei jugendlichen und erwachsenen Lernenden hingegen sollte der Bildungsanspruch einer BNE - also die Förderung von Emanzipation und Mündigkeit der Menschen (Klafki, 1996) - im Vordergrund stehen. Das heißt wiederum aber nicht, dass bei jugendlichen und erwachsenen Lernenden ESD 1 grundsätzlich ohne Bedeutung wäre. Pusch (2020, S. 136) charakterisiert politische Erziehung als einen pädagogischen Prozess, „,in dem politische Orientierungen zugemutet werden“ und hebt hervor, dass Erziehung die Möglichkeit impliziere, „, dass Menschen diese Zumutung zurückweisen können, wodurch die "Selbsttätigkeit des zu Erziehenden' (Schluss, 2007) zur Voraussetzung von Erziehung wird" (Pusch, 2020, S. 136). Und in diesem Sinne sollte BNE z. B. durchaus Lernenden die Aussage zumuten, dass eine nachhaltige Entwicklung in Deutschland als Ziel im Grundgesetz (Artikel 20a) verankert ist und ihre grundsätzliche Relevanz damit nicht zur Diskussion steht. Wohingegen die konkrete Ausgestaltung einer nachhaltigen Entwicklung offen ist und damit Gegenstand von (politischer) Bildung sein kann. Auch wenn in den folgenden Überlegungen BNE vor allem als (Allgemein-)Bildung charakterisiert wird, verfolgt sie also durchaus auch Erziehungsziele.

Der Bildungsanspruch einer BNE spiegelt sich deutlich in ihrem Ziel der Förderung von Schlüsselkompetenzen (d. h. fächer- und kontextübergreifenden Kompetenzen; Rychen, 2003; Weinert, 2001) wieder, die Individuen zu einer aktiven Gestaltung des Prozesses einer nachhaltigen Entwicklung befähigen, wie im Folgenden ausgeführt werden soll.

\section{Kompetenzerwerb im Rahmen von BNE}

Hinsichtlich des durch eine BNE zu erreichenden Kompetenzerwerbs wird in Deutschland häufig Bezug auf das Konzept der Gestaltungskompetenz genommen: „Gestaltungskompetenz bezeichnet die Fähigkeit, Probleme nicht nachhaltiger Entwicklungen erkennen und Wissen über nachhaltige Entwicklung wirksam anwenden zu können“ (de Haan, 2008, S. 12). Sie umfasst zwölf Teilkompetenzen, u. a. die Kompetenz zur Perspektivübernahme, die Kompetenz zur Antizipation und die Kompetenz zur Disziplinen übergreifenden Erkenntnisgewinnung (de Haan, 2008).

Zudem findet das im ,Orientierungsrahmen für den Lernbereich Globale Entwicklung im Rahmen einer Bildung für nachhaltige Entwicklung' (KMK \& BMZ, 2015) formulierte Kompetenzkonzept breite Anwendung. Dieses benennt Kernkompetenzen des Lernbereichs Globale Entwicklung in den drei Bereichen Erkennen, Bewerten und Handeln (für Tabelle 2 siehe Seite 9).

Auch auf internationaler Ebene findet eine intensive Auseinandersetzung mit der Kompetenzförderung durch BNE statt (Brundiers et al., 2021; Dlouhá, Heras, Mulà, Perez Salgado \& Henderson, 2019; Rieckmann, 2018; Lozano R., Merrill, Sammalisto, Ceulemans \& Lozano F., 2017; UNESCO, 2017; Wiek et al., 
2016; Mogensen \& Schnack, 2010). Derzeit werden im internationalen BNE-Diskurs die folgenden acht Nachhaltigkeitskompetenzen als besonders relevant angesehen (Rieckmann, 2018; UNESCO, 2017; Brundiers et al., 2021):

- Kompetenz zum Vernetzten Denken: die Fähigkeiten, Zusammenhänge zu erkennen und zu verstehen; komplexe Systeme zu analysieren; zu überlegen, wie Systeme in verschiedene Domänen und verschiedene Skalen eingebettet sind; und mit Unsicherheit umzugehen

- Kompetenz zum Vorausschauenden Denken: die Fähigkeiten, multiple (mögliche, wahrscheinliche und wünschenswerte) Zukünfte zu verstehen und zu bewerten; eigene Visionen für die Zukunft zu schaffen; das Vorsorgeprinzip anzuwenden; die Konsequenzen von Handlungen zu beurteilen; und mit Risiken und Veränderungen umzugehen

- Normative Kompetenz: die Fähigkeiten, die Normen und Werte zu verstehen und zu reflektieren, die den eigenen Handlungen zugrunde liegen; und Nachhaltigkeitswerte, Prinzipien und Ziele im Kontext von Interessenkonflikten und Trade-Offs, unsicheren Kenntnissen und Widersprüchen zu verhandeln

- Strategische Kompetenz: die Fähigkeiten zur kollektiven Entwicklung und Umsetzung innovativer Maßnahmen, die Nachhaltigkeit auf lokaler Ebene und darüber hinaus voranbringen

- Kooperationskompetenz: die Fähigkeiten, von anderen zu lernen; die Bedürfnisse, Perspektiven und Handlungen anderer zu verstehen und zu respektieren (Empathie), andere zu verstehen, eine Beziehung zu ihnen aufzubauen und für sie empfindsam zu sein (empathische Führung); mit Konflikten in einer Gruppe umzugehen; und eine kollaborative und partizipative Problemlösung zu ermöglichen

- Kompetenz zum Kritischen Denken: die Fähigkeit, Normen, Praktiken und Meinungen zu hinterfragen; die eigenen Werte, Wahrnehmungen und Handlungen zu reflektieren; und sich im Nachhaltigkeitsdiskurs zu positionieren

- Selbstkompetenz: die Fähigkeit, über die eigene Rolle in der lokalen Gemeinschaft und (globalen) Gesellschaft nachzudenken; kontinuierlich seine Handlungen zu bewerten und sich weiter zu motivieren; und sich mit den eigenen Gefühlen und Wünschen auseinanderzusetzen

- Integrierte Problemlösekompetenz: die übergreifende Fähigkeit, unterschiedliche Problemlösungsrahmen für komplexe Nachhaltigkeitsprobleme anzuwenden und passfähige, inklusive und gerechte Lösungsmöglichkeiten zu entwickeln, die eine nachhaltige Entwicklung fördern und die oben genannten Kompetenzen integrieren

\begin{tabular}{|l|l|l|}
\hline Erkennen & Bewerten & Handeln \\
\hline - Informationsbeschaffung & - Perspektivenwechsel & - Solidarität und \\
und -verarbeitung & Mitverantwortung \\
- Erkennen von Vielfalt & - Kritische Reflexion & - Verständigung und \\
- Analyse des Globalen Wandels & und Stellungnahme & Konfliktlösung \\
- Unterscheidung von & - Beurteilen von & - Handlungsfähigkeit im \\
Handlungsebenen & Entwicklungsmaßnahmen & globalen Wandel \\
& & - Partizipation und \\
& & Mitgestaltung \\
\hline
\end{tabular}

Tab. 2: Kernkompetenzen des Lernbereichs Globale Entwicklung (KMK \& BMZ, 2015, S. 96f.)

\section{BNE als Allgemeinbildung im Sinne Klafkis}

BNE hat also die Förderung von Kompetenzen zum Ziel, „, die eine zukunftsweisende und eigenverantwortliche Mitgestaltung einer nachhaltigen Entwicklung ermöglichen" (Michelsen, 2009, S. 84). Damit werden deutliche Parallelen zu Klafkis Bildungstheorie (Klafki, 2007, 1996) erkennbar. Auch im BNE-Konzept wird Bildung als Instrument zur gesellschaftlichen Entwicklung und Wahrnehmung von Verände- 
rungen und Widersprüchen innerhalb der Gesellschaft verstanden. Durch die Entwicklung von Nachhaltigkeitskompetenzen fördert BNE - ganz im Sinne Klafkis (2007; 1996) - die Emanzipation, Mündigkeit und Verantwortung des Menschen. Ebenso kann BNE als kritisch-konstruktive Bildung im Sinne Klafkis $(2007 ; 1996)$ verstanden werden. Denn durch die Entwicklung der genannten Kompetenzen befähigt sie zu wachsender Selbstbestimmung, Mitbestimmung und Solidarität (kritisch), und sie weist ein Handlungs-, Gestaltungs- und Veränderungsinteresse (konstruktiv) auf. Die genannten nachhaltigkeitsrelevanten Schlüsselkompetenzen setzen die Lernenden in die Lage, zur Bearbeitung aktuell diskutierter gesellschaftlicher Handlungs- und Gestaltungsfelder beizutragen, wie sie u. a. in den Sustainable Development Goals (SDGs) beschrieben werden, die mit Klafki (2007) als epochaltypische Schlüsselprobleme verstanden werden können (Stein, 2017). Es geht darum, Lernenden eine Auseinandersetzung mit gesellschaftlichen Schlüsselthemen zu ermöglichen und sie damit auf heutige und zukünftige Problemlösungen vorzubereiten (Overwien, 2013; Gräsel et al., 2012). Sie lernen, sich selbstständig mit den Schlüsselproblemen und "grundlegenden Lebensbereichen der globalisierten Welt" (Stein, 2017, S. 67) auseinanderzusetzen. Hier lassen sich auch Bezüge zu Klafkis Konzept kategorialer Bildung (Klafki, 2007) herstellen, die eine dialektische Verbindung zwischen dem Erschließen von Bildungsinhalten (materiale Bildung) und den sich bildenden Subjekten (formale Bildung) beschreibt: „Inhalte der objektiven Welt werden in subjektive Erfahrungen und Fertigkeiten des Individuums umgesetzt" (Stein, 2017, S. 111). Insgesamt lässt sich feststellen, dass BNE als eine aktualisierte Konzeption von Allgemeinbildung im Sinne Klafkis betrachtet werden kann.

\section{BNE als transformative Bildung}

BNE beschränkt sich jedoch nicht auf die Entwicklung von Kompetenzen; sie zielt als transformative Bildung auch auf die „Transformation des individuellen ,Selbst- und Weltverhältnisses' (Koller, 2011, S. 16) im Sinne einer globalen Perspektive“ (Scheunpflug, 2019, S. 66) und damit auf die Veränderung von Einstellungen, Werten, Paradigmen und Weltanschauungen (Balsiger et al., 2017; Sterling, 2011).

BNE stellt einen direkten Zusammenhang zwischen individuellen Veränderungen und gesellschaftlichem Wandel her und kann somit als transformative Bildung betrachtet werden (Scheunpflug, 2019; Koller, 2012). Ein ,conceptual change', d. h. die Veränderung von grundlegenden Orientierungen (Einstellungen, Werten, Paradigmen und Weltanschauungen) (Scheunpflug, 2019, S. 65), kann und soll durch BNE gefördert werden. Mit der Perspektive einer nachhaltigen Entwicklung fokussiert BNE die Reflexion und die Veränderung des „gesamte[n] Verhältnis des Subjekts zur Welt, zu anderen und zu sich selber" (Koller, 2012, S. 9). Mit BNE wird eine Antwort auf die Frage gegeben, wie ein Anpassungsprozess an neuartige Problemlagen (Koller, 2012) befördert werden kann.

Während transformatives Lernen darauf abzielt, Lernende zu befähigen, die Art und Weise zu hinterfragen und zu verändern, wie sie die Welt sehen und über sie denken (Slavich \& Zimbardo, 2012; Mezirow, 2000), geht das verwandte Konzept des transgressiven Lernens (Lotz-Sisitka, Wals, Kronlid \& McGarry, 2015) noch einen Schritt weiter: Es betont, dass das Lernen in der BNE den Status quo überwinden und die Lernenden für disruptives Denken und die Ko-Kreation neuen Wissens vorbereiten sollte.

BNE im Sinne von emanzipatorischer Bildung (ESD 2) geht es zwar nicht darum, vorgegebene Einstellungen, Werten, Paradigmen und Weltanschauungen zu vermitteln. Gleichwohl orientiert sich BNE an den Ideen der intra- und intergenerationellen Gerechtigkeit (de Haan, 2008). Sie verfolgt vor diesem Hintergrund stets das wertbezogene Ziel, zu einer "Sensibilisierung für eine Überlebensverantwortung" (Mokrosch, 2008, S. 38) beizutragen. Zugleich ist es ein pädagogisches Ziel einer BNE, eine Auseinandersetzung mit Werthaltungen im Sinne einer „Werteklärung“ (Mokrosch, 2008, S. 36) zu ermöglichen, die mit dem Leitbild einer nachhaltigen Entwicklung verbunden sind: insbesondere in Bezug auf den Erhalt der natürlichen Lebensgrundlagen, Menschenwürde und Gerechtigkeit (Stoltenberg, 2009). Eine 
so verstandene BNE kann zu einem „Wertewandel zur Nachhaltigkeit“ (WBGU, 2011) beitragen, ohne Lernende zu bevormunden oder zu überwältigen. Im Gegenteil unterstützt sie die Entwicklung einer reflexiven Kompetenz. Oder anders gesagt: „Transformative learning must not be used to instrumentalize learners but to empower them for autonomous critical action” (Balsiger et al., 2017, S. 359).

Von BNE ist daher ein Beitrag zu einem kritischen Wertediskurs gleichermaßen zu erwarten und einzufordern (Rieckmann \& Schank, 2016; Rieckmann, Fischer \& Richter, 2014). Sie kann und soll Anregungen geben, die eigenen Werte zu reflektieren und in der Wertedebatte auf dem Weg zu einer nachhaltigen Entwicklung Stellung zu beziehen (Balsiger et al., 2017; Rieckmann \& Schank, 2016). Ihr Potenzial liegt somit auch darin, den Wertehorizont der Lernenden zu erweitern. So können z. B. die lateinamerikanischen Diskurse zum Buen Vivir (Gutes Leben) und zu den Rechten der Natur in die Wertedebatte einbezogen werden (Rieckmann, 2017). Damit können eurozentrische Denkweisen erweitert und kritisch reflektiert werden.

Um im Rahmen von BNE tatsächlich Prozesse transformativer Bildung zu befördern, braucht es Lernumgebungen, in denen die Lernenden sich ihrer eigenen und der Annahmen anderer bewusstwerden und diese kritisch reflektieren können (Mezirow, 1997) und in denen sie ermutigt werden, die Bezugsrahmen auf kritische, de-konstruierende und transgressive Weise zu reflektieren, um wirklich transformative Lernprozesse anzuregen, die zu ,conceptual change' führen (Rodríguez Aboytes \& Barth, 2020; Lotz-Sisitka et al., 2015; Sterling, 2011).

Für eine Transformation im Sinne einer nachhaltigen Entwicklung bedarf es aber auch struktureller und institutioneller Veränderungen (WBGU, 2011). Daher sollte BNE nicht nur die Ebene individuellen Verhaltens - und den dafür nötigen Wissens- und Kompetenzerwerb sowie eine entsprechende Werteorientierung - fokussieren, sondern auch die Frage nach den Strukturen, nach der „Großen Transformation“ (WBGU, 2011) aufwerfen. BNE sollte zur (politischen) Bildung von Nachhaltigkeitsbürger*innen beitragen, die - im Sinne transgressiven Lernens - befähigt sind, die bestehenden Strukturen in Frage zu stellen, über diese hinauszudenken und somit zur strukturellen und institutionellen Transformation beitragen zu können (Rieckmann, 2020; 2017; Balsiger et al., 2017; Rieckmann \& Schank, 2016).

\section{Bezüge von BNE zu Krügers Reflexiver Erziehungswissenschaft}

Des Weiteren lassen sich Bezüge zu Krügers Reflexiver Erziehungswissenschaft (Krüger, 2012) herstellen. Krüger beschreibt als deren wesentliche Ziele die Auseinandersetzung mit globalen Gefährdungslagen und Risiken (reflexive Modernisierung), die Förderung von (posttraditionaler) Solidarität und die Verbesserung der pädagogischen Praxis (Krüger, 2012, S. 166ff., 247ff.). Zum Erreichen dieser Ziele kann eine BNE Beiträge leisten: zu den beiden erstgenannten v. a. durch die Entwicklung der Schlüsselkompetenzen; zum letzten insbesondere durch ihre handlungsorientierte, transformative Pädagogik (Rieckmann, 2018; UNESCO, 2017). Gleichzeitig ergeben sich aber auch Anregungen aus der reflexiven Erziehungswissenschaft für das BNE-Konzept. So verweist die Theorie reflexiver Modernisierung z. B. darauf, dass die „ungewollten und unabschätzbaren externen und internen Nebenfolgen industriegesellschaftlicher Modernisierung [...] nicht nur den Erhalt der Natur gefährden, sondern auch alle gesellschaftlichen Institutionen der klassischen Industriemoderne durcheinanderwirbeln" (Krüger, 2012, S. 250). Sie beleuchtet die stattfindenden tiefgreifenden gesellschaftlichen Wandlungsprozesse, übt Kritik an der Gesellschaft der ,Moderne' und verdeutlicht damit die Notwendigkeit struktureller und institutioneller Transformation. Zudem soll gemäß Krügers Reflexiver Erziehungswissenschaft Bildung „für den Umgang mit Vielfalt und die soziale Wertschätzung und Anerkennung der Heterogenität des Anderen, d. h. auch für die Anerkennung von Geschlechterdifferenzen und kulturellen Differenzen, sensibilisieren" (Krüger, 2012, S. 256). Hieraus können sich Hinweise für eine notwendige und noch ausstehende inklusivere Gestaltung von BNE (Vierbuchen \& Rieckmann, 2020) ergeben. 


\section{Parallelen einer BNE zu Freires Befreiungspädagogik}

Es ist bereits deutlich geworden, dass BNE zur Förderung von Emanzipation und Mündigkeit der Lernenden sowie auch zu transformativem und transgressivem Lernen beiträgt. Damit knüpft sie auch an die Ideen der Befreiungspädagogik Freires (2008) an (Misiaszek, 2020).

Denn BNE schafft ein Bewusstsein für Ungerechtigkeiten, Unterdrückung und Diskriminierungen, und befähigt die Lernenden zur Überwindung der bestehenden Verhältnisse und für eine gesellschaftliche Veränderung. Die Lernenden „lernen die Welt nicht als statische Wirklichkeit, sondern als eine Wirklichkeit im Prozess sehen, in der Umwandlung" (Freire, 1973, S. 67). Sie werden in die Lage versetzt, als Subjekte zu handeln, wobei Aktion und Reflexion - als wesentliche Elemente einer Kompetenzentwicklung - im Wechselspiel stehen.

Auch die didaktischen Zugänge der Befreiungspädagogik, wie z. B. ein dialogisch gestalteter Lernprozess mit einer „horizontale[n] Beziehung zwischen [den beteiligten] Personen” (Freire, 1974, S. 61, zitiert nach Overwien, 2003, S. 3) sowie die partizipative Erforschung des Lebensumfeldes und die damit einhergehende Bewusstwerdung über die soziale und politische Lage der Lernenden (Freire, 2008; Stöger, 2017), weisen Parallelen zu den didaktischen Prinzipien einer BNE auf und können für diese fruchtbar gemacht werden (Souza, Wals \& Jacobi, 2019). Die Freireanische Pädagogik kann eine hilfreiche und effektive Methode sein, um eine auf kritischer Selbstreflexion basierende Bildungspraxis als „starting point for creating pedagogies for positive change“ (Kertyzia, 2020, S. 150) zu etablieren.

\section{Schlussfolgerungen}

BNE ist ein relativ junges Bildungskonzept, das sich in der Erziehungswissenschaft wie auch in der pädagogischen Praxis in den letzten 20 Jahren etabliert hat. Mit den Ausführungen in diesem Artikel ist allerdings deutlich geworden, dass BNE eine hohe Anschlussfähigkeit an auch ältere erziehungswissenschaftliche Diskurse und Theorien aufweist. BNE lässt sich erstens besser verstehen und verorten, wenn sie auf Theorien der Erziehung und Bildung Bezug nimmt. Zweitens lässt sich BNE als eine aktualisierte Konzeption von Allgemeinbildung im Sinne Klafkis betrachten. Drittens sind Theorien zur transformativen Bildung sehr nützlich, um die im Rahmen einer BNE angestrebte Werte-Bildung zu beschreiben. Viertens ergeben sich aus Krügers Reflexiver Erziehungswissenschaft Anregungen für eine Auseinandersetzung mit der Notwendigkeit struktureller und institutioneller Transformation und für eine inklusivere Gestaltung von BNE. Fünftens weist BNE Parallelen zu Freires Befreiungspädagogik auf, und sie könnte von einer Auseinandersetzung mit den didaktischen Zugängen der Befreiungspädagogik profitieren.

BNE hat in den letzten Jahren nicht nur in der pädagogischen Praxis an Bedeutung gewonnen, sondern wird auch im erziehungswissenschaftlichen Diskurs stärker wahrgenommen und aufgegriffen. Die BNE-Community selbst hat sich bisher aber nur in Ansätzen an der Weiterentwicklung von Theorien der Erziehung und Bildung beteiligt. Dass hier durchaus relevante Beiträge möglich wären, konnte dieser Beitrag nur andeuten. Es wäre wünschenswert, dass die hier aufgezeigten Stränge weiterverfolgt und vertiefend bearbeitet werden.

\section{Literaturverzeichnis}

Balsiger, Jörg; Förster, Ruth; Mader, Clemens; Nagel, Ueli; Sironi, Helene; Wilhelm, Sandra \& Zimmermann, Anne B. (2017). Transformative Learning and Education for Sustainable Development. GAIA - Ecological Perspectives for Science and Society, 26(4), 357-359. https://doi.org/10.14512/gaia.26.4.15

Barth, Matthias (2016). Forschung in der Bildung für nachhaltige Entwicklung: Entstehung und Verortung eines Forschungszweiges. In Matthias Barth \& Marco Rieckmann (Hg.), Empirische Forschung 
zur Bildung für nachhaltige Entwicklung - Themen, Methoden und Trends (S. 37-49). Opladen: Barbara Budrich.

Brezinka, Wolfgang (1990). Grundbegriffe der Erziehungswissenschaft. Analyse, Kritik, Vorschläge. München: Ernst Reinhardt.

Brundiers, Katja; Barth, Matthias; Cebrián, Gisela; Cohen, Matthew; Diaz, Liliana; Doucette-Remington, Sonya; Dripps, Weston; Habron, Geoffrey; Harré, Niki; Jarchow, Meghann; Losch, Kealalokahi; Michel, Jessica; Mochizuki, Yoko; Rieckmann, Marco; Parnell, Roderic; Walker, Peter \& Zint, Michaela (2021). Key competencies in sustainability in higher education - toward an agreed-upon reference framework. Sustainability Science, 16(1), 13-29. https://doi.org/10.1007/s11625-020-00838-2

de Haan, Gerhard (2008). Gestaltungskompetenz als Kompetenzkonzept der Bildung für nachhaltige Entwicklung. In Inka Bormann \& Gerhard de Haan (Hg.), Kompetenzen der Bildung für nachhaltige Entwicklung. Operationalisierung, Messung, Rahmenbedingungen, Befunde (S. 23-43). Wiesbaden: Verlag für Sozialwissenschaften.

de Haan, Gerhard; Kamp, Georg; Lerch, Achim; Martignon, Laura; Müller-Christ, Georg \& Nutzinger, Hans G. (Hg.) (2008). Nachhaltigkeit und Gerechtigkeit. Grundlagen und schulpraktische Konsequenzen. Berlin: Springer.

Dlouhá, Jana; Heras, Raquel; Mulà, Ingrid; Perez Salgado, Francisca \& Henderson, Laura (2019). Competences to Address SDGs in Higher Education - A Reflection on the Equilibrium between Systemic and Personal Approaches to Achieve Transformative Action. Sustainability, 11(13). 3664. https://doi.org/10.3390/su11133664

Freire, Paulo (1973). Pädagogik der Unterdrückten. Bildung als Praxis der Freiheit. Reinbek: Rowohlt.

Freire, Paulo (2008). Pädagogik der Autonomie. Notwendiges Wissen für die Bildungspraxis. Münster: Waxmann.

Gräsel, Cornelia; Bormann, Inka; Schütte, Kerstin; Trempler, Kati; Fischbach, Robert \& Asseburg, Regine (2012). Perspektiven der Forschung im Bereich Bildung für nachhaltige Entwicklung. In Bundesministerium für Bildung und Forschung [BMBF] (Hg.), Bildung für nachhaltige Entwicklung. Beiträge der Bildungsforschung, Band 39 (S. 7-24). Berlin: Bundesministerium für Bildung und Forschung.

Gramelt, Katja (2010). Der Anti-Bias-Ansatz. Zu Konzept und Praxis einer Pädagogik für den Umgang mit (kultureller) Vielfalt. Wiesbaden: Verlag für Sozialwissenschaften.

Kertyzia, Heather (2020). Who we are matters: An autoethnography of Global Citizenship Education in intersectionally diverse contexts. Tertium Comparationis: Journal für International und Interkulturell Vergleichende Erziehungswissenschaft, 26(2), 146-151. https://www.waxmann.com/index.php?eID=download\&id artikel=ART104542\&uid=frei

Klafki, Wolfgang (1996). Neue Studien zur Bildungstheorie und Didaktik. Zeitgemäße Allgemeinbildung und kritisch-konstruktive Didaktik (5. Auflage). Weinheim: Beltz.

Klafki, Wolfgang (2007). Konturen eines neuen Allgemeinbildungskonzepts. In Wolfgang Klafki (Hg.), Neue Studien zur Bildungstheorie und Didaktik. Zeitgemäße Allgemeinbildung und kritisch-konstruktive Didaktik (6. Auflage) (S. 43-81). Weinheim: Beltz.

Kultusministerkonferenz [KMK] \& Bundesministerium für wirtschaftliche Zusammenarbeit und Entwicklung [BMZ] (2015). Orientierungsrahmen für den Lernbereich Globale Entwicklung im Rahmen einer Bildung für nachhaltige Entwicklung. http://www.kmk.org/fileadmin/veroeffentlichungen beschluesse/2015/2015 06 00-Orientierungsrahmen-Globale-Entwicklung.pdf

Koller, Hans-Christoph (2012). Bildung anders denken. Einführung in die Theorie transformatorischer Bildungsprozesse. Stuttgart: Kohlhammer.

Krüger, Heinz-Hermann (2012). Einführung in Theorien und Methoden der Erziehungswissenschaft. Opladen: Barbara Budrich.

Lotz-Sisitka, Heila; Wals, Arjen E. J.; Kronlid, David \& McGarry, Dylan (2015). Transformative, transgressive social learning: rethinking higher education pedagogy in times of systemic global dys- 
function. Current Opinion in Environmental Sustainability, 16, 73-80. https://doi.org/10.1016/j.cosust.$\underline{2015.07 .018}$

Lozano, Rodrigo; Merrill, Michelle Y.; Sammalisto, Kaisu; Ceulemans, Kim \& Lozano, Francisco J. (2017). Connecting Competences and Pedagogical Approaches for Sustainable Development in Higher Education: A Literature Review and Framework Proposal. Sustainability, 9(10), 1889. https://doi.org/10.3390/su9101889

Mezirow, Jack (1997). Transformative Learning: Theory to Practice. New Directions for Adult \& Continuing Education, 74, 5-12, https://doi.org/10.1002/ace.7401

Mezirow, Jack (2000). Learning as transformation: critical perspectives on a theory in progress. San Francisco: Jossey-Bass.

Michelsen, Gerd (2009). Kompetenzen und Bildung für nachhaltige Entwicklung. In Bernd Overwien \& Hanns-Fred Rathenow (Hg.), Globalisierung fordert politische Bildung: Politisches Lernen im globalen Kontext (S. 75-86). Opladen: Barbara Budrich.

Michelsen, Gerd \& Fischer, Daniel (2015). Bildung für nachhaltige Entwicklung. Wiesbaden: Hessische Landeszentrale für politische Bildung.

Misiaszek, Greg W. (2020). Ecopedagogy: teaching critical literacies of 'development', 'sustainability', and 'sustainable development'. Teaching in Higher Education, 25(5), 615-632. https://doi.org/10.1080/$\underline{13562517.2019 .1586668}$

Mogensen, Finn \& Schnack, Karsten (2010). The action competence approach and the 'new' discourses of education for sustainable development, competence and quality criteria. Environmental Education Research, 16(1), 59-74. https://doi.org/10.1080/13504620903504032

Mokrosch, Reinhold (2008). Zum Verständnis von Werte-Erziehung: Aktuelle Modelle für die Schule. In Reinhold Mokrosch \& Arnim Regenbogen (Hg.), Werte-Erziehung und Schule. Ein Handbuch für Unterrichtende (S. 32-40). Göttingen: Vandenhoeck \& Ruprecht.

Mulder, Karel (2014). Strategic competencies, critically important for Sustainable Development. Journal of Cleaner Production, 78, 243-248. https://doi.org/10.1016/j.jclepro.2014.03.098

Müller, Ulrich (2000). Der Mensch im Mittelpunk. Bildung für nachhaltige Entwicklung benötigt die Klärung des Bildungsbegriffs. Politische Ökologie, Sonderheft 12, 8-11. https://www.oekom.de/ uploads media/files/poe sohe 122000 ulrich mu ller 041415.pdf

Overwien, Bernd (2003). Das lernende Subjekt als Ausgangspunkt - Befreiungspädagogik und informelles Lernen. In Wolfgang Wittwer \& Steffen Kirchhof (Hg.), Informelles Lernen und Weiterbildung: Neue Wege zur Kompetenzentwicklung (S.43-64). München: Luchterhand. https://www.globaleslernen.de/sites/default/files/files/link-elements/Overwien\%20lernendes\%20Subjekt.pdf

Overwien, Bernd (2013). Kompetenzmodelle im Lernbereich "Globale Entwicklung“ - Bildung für nachhaltige Entwicklung. In Bernd Overwien \& Horst Rode (Hg.), Bildung für nachhaltige Entwicklung. Lebenslanges Lernen, Kompetenz und gesellschaftliche Teilhabe (S. 13-34). Opladen: Barbara Budrich.

Pusch, Barbara (2020). Bildung für nachhaltige Entwicklung in Orientierungs- und Wertekursen? Ein österreichisch-deutscher Kursbuchvergleich. Österreichisches Religionspädagogisches Forum, 28(2), 132-148. https://doi.org/10.25364/10.28:2020.2.9

Rieckmann, Marco (2016). Bildung für nachhaltige Entwicklung - Konzeptionelle Grundlagen und Stand der Implementierung. In Martin K. W. Schweer (Hg.), Bildung für nachhaltige Entwicklung in pädagogischen Handlungsfeldern. Grundlagen, Verankerung und Methodik in ausgewählten Lehr-Lern-Kontexten (S. 11-32). Frankfurt am Main: PL Academic Research.

Rieckmann, Marco (2017). Bildung für nachhaltige Entwicklung in der Großen Transformation - Neue Perspektiven aus den Buen Vivir- und Postwachstumsdiskursen. In Oliver Emde, Uwe Jakubczyk, Bernd Kappes \& Bernd Overwien (Hg.), Mit Bildung die Welt verändern? Globales Lernen für eine nachhaltige Entwicklung (S. 147-159). Opladen: Barbara Budrich. 
Rieckmann, Marco (2018). Learning to transform the world: key competencies in ESD. In Alexander Leicht, Julia Heiss \& Won Jung Byun (Eds.), Education on the move. Issues and trends in education for sustainable development (S. 39-59). Paris: UNESCO.

Rieckmann, Marco (2020). Emancipatory and Transformative Global Citizenship Education in Formal and Informal Settings: Empowering Learners to Change Structures. Tertium Comparationis: Journal für International und Interkulturell Vergleichende Erziehungswissenschaft, 26(2), 174-186. https://www.waxmann.com/index.php?eID=download\&id artikel=ART104545\&uid=frei

Rieckmann, Marco \& Schank, Christoph (2016). Sozioökonomisch fundierte Bildung für nachhaltige Entwicklung - Kompetenzentwicklung und Werteorientierungen zwischen individueller Verantwortung und struktureller Transformation. SOCIENCE - Journal of Science-Society Interfaces, 1(1), 6579 .

Rieckmann, Marco; Fischer, Daniel \& Richter, Sonja (2014). Nachhaltige Ernährung im Wertediskurs. In Christoph Schank, Kristin Vorbohle \& Jan Hendrik Quandt (Hg.), Perspektive Nahrungsmittelethik (S. 29-58). München: Rainer Hampp Verlag.

Rodríguez Aboytes, Jorge G. \& Barth, Matthias (2020). Transformative learning in the field of sustainability: a systematic literature review (1999-2019). International Journal of Sustainability in Higher Education, 21(5), 993-1013. https://doi.org/10.1108/IISHE-05-2019-0168

Rychen, Dominique S. (2003). Key competencies: Meeting important challenges in life. In Dominique S. Rychen \& Laura Hersh Salganik (Eds.), Key competencies for a successful life and well-functioning society (pp. 63-107). Bern: Hogrefe und Huber.

Scheunpflug, Annette (2019). Transformatives Globales Lernen - eine Grundlegung in didaktischer Absicht. In Gregor Lang-Wojtasik (Hg.), Bildung für eine Welt in Transformation. Global Citizenship Education als Chance für die Weltgesellschaft (S. 63-74). Opladen: Barbara Budrich.

Seitz, Klaus (2002). Bildung in der Weltgesellschaft. Gesellschaftstheoretische Grundlagen Globalen Lernens. Frankfurt am Main: Brandes und Apsel Verlag.

Simojoki, Henrik (2012). Globalisierte Religion: Ausgangspunkte, Maßstäbe und Perspektiven religiöser Bildung in der Weltgesellschaft (Praktische Theologie in Geschichte und Gegenwart 12). Tübingen: Mohr Siebeck.

Slavich, George M. \& Zimbardo, Philip G. (2012). Transformational Teaching: Theoretical Underpinnings, Basic Principles, and Core Methods. Educational Psychology Review, 24, 569-608. https://doi.org/10.1007/s10648-012-9199-6

Souza, Daniele T.; Wals, Arjen E. J. \& Jacobi, Pedro R. (2019). Learning-based transformations towards sustainability: a relational approach based on Humberto Maturana and Paulo Freire. Environmental Education Research, 25(11), 1605-1619. https://doi.org/10.1080/13504622.2019.1641183

Stein, Margit (2017). Allgemeine Pädagogik. (3., überarbeitete Auflage). München: Ernst Reinhardt Verlag. Steffen, Will; Richardson, Katherine; Rockström, Johan; Cornell, Sarah E.; Fetzer, Ingo; Bennett, Elena M.; Biggs, Reinette; Carpenter, Stephen R.; Vries, Wim de; Wit, Cynthia A. de; Folke, Carl; Gerten, Dieter; Heinke, Jens; Mace, Georgina M.; Persson, Linn M.; Ramanathan; Veerabhadran; Reyers, Belinda \& Sörlin, Sverker (2015). Planetary boundaries: Guiding human development on a changing planet. Science, 347(6223). https://doi.org/10.1126/science.1259855

Sterling, Stephen (2011). Transformative learning and sustainability: sketching the conceptual ground. Learning and Teaching in Higher Education, 5, 17-33.

Stöger, Peter (2017). Paulo Freire und seine Befreiungspädagogik - Essayistische Anmerkungen. In Laura Burkhardt, Helmwart Hierdeis \& Theo Hug (Hg.), Kritische Lektionen (S. 207-218). Innsbruck: innsbruck university press.

Stoltenberg, Ute (2009). Mensch und Wald. Theorie und Praxis einer Bildung für nachhaltige Entwicklung am Beispiel des Themenfeldes Wald. München: oekom Verlag.

UN Environment (Ed.) (2019). Global Environment Outlook GEO-6: Healthy Planet, Healthy People. Cambridge: Cambridge University Press. https://www.unep.org/resources/global-environment-outlook-6 
United Nations (2015). Transforming our world: the 2030 Agenda for Sustainable Development. Resolution adopted by the General Assembly on 25 September 2015. http://www.un.org/ga/search/view doc.asp?symbol=A/RES/70/1\&Lang $=\mathrm{E}$

United Nations Educational, Scientific and Cultural Organization [UNESCO] (2017). Education for Sustainable Development Goals. Learning Objectives. Paris: UNESCO. http://unesdoc.unesco.org/imagesL0024/002474/247444e.pdf

United Nations Educational, Scientific and Cultural Organization [UNESCO] (2021). Berliner Erklärung zur Bildung für nachhaltige Entwicklung. https://www.unesco.de/sites/default/files/2021-05/Berliner\%20Erk1\%C3\%A4rung\%20f\%C3\%BCr\%20BNE.pdf

Vare, Paul \& Scott, William (2007). Learning for a Change: Exploring the Relationship Between Education and Sustainable Development. Journal of Education for Sustainable Development, 1(2), 191-198. https://doi.org/10.1177/097340820700100209

Vierbuchen, Marie-Christine \& Rieckmann, Marco (2020). Bildung für nachhaltige Entwicklung und inklusive Bildung - Grundlagen, Konzepte und Potenziale. ZEP - Zeitschrift für internationale Bildungsforschung und Entwicklungspädagogik, 43(1), 4-10. https://doi.org/10.31244/zep.2020.01.02

Wals, Arjen E. J. (2011). Learning Our Way to Sustainability. Journal of Education for Sustainable Development, 5(2), 177-186. https://doi.org/10.1177/097340821100500208

Wissenschaftlicher Beirat der Bundesregierung Globale Umweltveränderungen [WBGU] (2011). Welt im Wandel: Gesellschaftsvertrag für eine große Transformation. https://issuu.com/wbgu/docs/wbgu jg2011? $=37591641 / 69400318$

Weinert, Franz Erich (2001). Concept of Competence: A Conceptual Clarification. In Dominique Simone Rychen \& Laura Hersh Salganik (Eds.), Defining and Selecting Key Competencies (pp. 45-65). Göttingen: Hogrefe und Huber.

Wiek, Arnim; Bernstein, Michael J.; Foley, Rider W.; Cohen, Matthew; Forrest, Nigel; Kuzdas, Christopher; Kay, Braden \& Withycombe Keeler, Lauren (2016). Operationalising competencies in higher education for sustainable development. In Matthias Barth, Gerd Michelsen, Marco Rieckmann \& Ian Thomas (Eds.), Routledge Handbook of Higher Education for Sustainable Development (pp. 241-260). London: Routledge.

World Bank (2020). Reversals of Fortune. Poverty and Shared Prosperity 2020. Washington DC: World Bank Publications. https://www.worldbank.org/en/publication/poverty-and-shared-prosperity 